\title{
Flow shear stabilization of rotating plasmas due to the Coriolis effect
}

\author{
J. W. Haverkort* and H. J. de Blank \\ FOM Institute DIFFER - Dutch Institute for Fundamental Energy Research, Association EURATOM/FOM, Trilateral Euregio Cluster
}

(Received 13 April 2012; revised manuscript received 29 June 2012; published 30 July 2012)

\begin{abstract}
A radially decreasing toroidal rotation frequency can have a stabilizing effect on nonaxisymmetric magnetohydrodynamic (MHD) instabilities. We show that this is a consequence of the Coriolis effect that induces a restoring pressure gradient force when plasma is perturbed radially. In a rotating cylindrical plasma, this Coriolis-pressure effect is canceled by the centrifugal effect responsible for the magnetorotational instability. In a magnetically confined toroidal plasma, a large aspect ratio expansion shows that only half of the effect is canceled. This analytical result is confirmed by numerical computations. When the plasma rotates faster toroidally in the core than near the edge, the effect can contribute to the formation of transport barriers by stabilizing MHD instabilities.
\end{abstract}

DOI: 10.1103/PhysRevE.86.016411

\section{INTRODUCTION}

Plasma flow in present-day tokamaks is primarily beneficial. Arguably most important is the suppressing effect flow shear has on turbulence, by tearing apart the turbulent eddies. The associated reduction in thermal transport greatly benefits the goal of commercially producing energy from nuclear fusion in a tokamak. Flow shear also plays a crucial role in explaining angular momentum losses and accretion rates in astrophysical plasmas through the axisymmetric magnetorotational instability (MRI) [1,2].

This paper concerns an effect of flow shear caused by the influence of the Coriolis effect in rotating plasmas. It arises through a spatial variation in the Doppler shifted mode frequency. This has been overlooked in the first two analyses of localized MHD modes [3,4]. In integrated form the effect first appeared in an analysis of the internal kink mode [5] where it was grouped with inertial terms and was denoted as "flutter." The effect also surfaced in more recent analyses [6,7]. In Ref. [7] a stability criterion is derived for modes localized in the $r$ direction, normal to the circular magnetic surfaces of a large aspect ratio tokamak plasma. For not too high rotation this result reads [7]

$$
\frac{r B_{0}^{2}}{4 R_{0}^{2}}\left(\frac{q^{\prime}}{q^{2}}\right)^{2}>-2 p^{\prime} \frac{1-q^{2}}{q^{2} R_{0}^{2}}+\frac{r}{4} \frac{1+2 q^{2}}{q^{2}} \rho \Omega^{\prime 2}+\rho^{2}\left(\frac{\Omega^{2}}{\rho}\right)^{\prime},
$$

with $\Omega$ the angular rotation frequency and $B_{0}$ the vacuum magnetic field at the major radius $R=R_{0}$. A prime denotes a radial $r$ derivative. The pressure $p$ and density $\rho$ should be averaged over a magnetic surface, or read $\rho_{0}$ and $p_{0}$ as defined in Eqs. (26) and (27) below. The safety factor $q$ is the number of toroidal turns per poloidal turn of a magnetic field line. The static part of Eq. (1) has a long history, dating back to the cylindrical result of Ref. [8], the arbitrary aspect ratio result of Ref. [9], and the large aspect ratio results of Refs. [10,11].

Writing

$$
\rho^{2}\left(\frac{\Omega^{2}}{\rho}\right)^{\prime}=2 \rho \Omega^{2^{\prime}}-\rho^{\prime} \Omega^{2}-\rho \Omega^{2^{\prime}}
$$

\footnotetext{
*Centrum Wiskunde, \& Informatica (CWI), P.O. Box 94079, Amsterdam, The Netherlands; j.w.haverkort@cwi.nl
}

PACS number(s): 52.55.Tn, 47.20.Qr, 52.35.Py, 95.30.Qd

the second term represents the centrifugal analog of the Rayleigh-Taylor instability of a heavy fluid on top of a lighter one. The final term of Eq. (2) is responsible for the MRI, but it is canceled by the two times larger first term that is the subject of this work. This Coriolis-pressure term ensures that a radially decreasing angular rotation frequency is stabilizing when the destabilizing Kelvin-Helmholtz term proportional to $\Omega^{\prime 2}$ is not too large. This is the case when

$$
\left|\frac{r \Omega^{\prime}}{\Omega}\right|<\frac{8 q^{2}}{1+2 q^{2}},
$$

which in a tokamak ranges from 2.67 in the core where $q=1$ to 4 near the edge where $q \gg 1$. First, we will investigate the effect of the Coriolis force on nonaxisymmetric modes in a cylindrical geometry, to find that its influence is exactly canceled by the MRI effect. Next, we perform a large aspect ratio expansion to show that in toroidal geometry only half of the effect is canceled by the MRI. Finally, we confirm this result with linear simulations showing that the effect can stabilize interchange instabilities in the low magnetic shear core region of a tokamak plasma.

\section{PRELIMINARIES}

We consider a rotating ideal MHD equilibrium in which the pressure force and centrifugal force are balanced by the Lorentz force and/or gravitational force

$$
\nabla p=\rho \Omega^{2} \mathbf{R}+(\nabla \times \mathbf{B}) \times \mathbf{B}-\rho \nabla \Phi .
$$

We assume axisymmetry and a rotation velocity $\mathbf{u}=R \Omega \hat{\mathbf{e}}_{\phi}$ in the azimuthal $\hat{\mathbf{e}}_{\phi}=R \nabla \phi$ direction of an $(R, z, \phi)$ coordinate system with $\hat{\mathbf{e}}_{z}=\nabla z$ the axis of symmetry. We investigate perturbations $\xi \propto e^{-i(n \phi+\omega t)}$ relative to the rotating plasma, described by the Frieman-Rotenberg equation $[5,12]$ (see Appendix A)

$$
-\rho \omega_{D}^{2} \boldsymbol{\xi}+2 i \rho \omega_{D} \Omega \hat{\mathbf{e}}_{z} \times \boldsymbol{\xi}=\mathbf{F}_{s}-\mathbf{R} \nabla \cdot\left(\rho \Omega^{2} \boldsymbol{\xi}\right),
$$

where $\omega_{D} \equiv \omega+n \Omega$ is the Doppler shifted frequency and $\mathbf{F}_{s}$ is the static ideal MHD force operator [13]

$$
\mathbf{F}_{s}=-\nabla \delta p+(\nabla \times \mathbf{B}) \times \delta \mathbf{B}+(\nabla \times \delta \mathbf{B}) \times \mathbf{B}-\delta \rho \nabla \Phi .
$$


The perturbed pressure, density, and magnetic field are given by $\delta p=-\gamma p \nabla \cdot \boldsymbol{\xi}-\boldsymbol{\xi} \cdot \nabla p, \delta \rho=-\nabla \cdot(\rho \boldsymbol{\xi})$, and $\delta \mathbf{B}=\nabla \times$ $(\boldsymbol{\xi} \times \mathbf{B})$, respectively.

In Eq. (5) we clearly recognize the second term as the Coriolis force, the fictitious force experienced by a plasma element moving with a velocity $i \omega_{D} \xi$ relative to the rotating background plasma.

\section{CYLINDRICAL ANALYSIS}

\section{A. Simplified analysis}

We first analyze a simple hydrodynamic cylindrical configuration in which the equilibrium quantities vary only with $R$. We include a gravitational field and no equilibrium magnetic field. The radial component of the force balance equation (4) then reads

$$
p^{\prime}=\rho\left(R \Omega^{2}-\Phi^{\prime}\right)
$$

where a prime now denotes an $R$ derivative. To simplify the analysis, we take the incompressible limit $\nabla \cdot \xi \rightarrow 0, \gamma \rightarrow \infty$ keeping $\gamma p \nabla \cdot \xi$ finite. We consider only nonaxisymmetric modes so that $n \neq 0$. The $\phi$ component of Eq. (5) then gives

$$
\delta p=-\frac{\rho \omega_{D}^{2}}{i n} R \xi_{\phi}-\frac{2 \rho \omega_{D} \Omega}{n} R \xi_{R} .
$$

Incompressibility $\nabla \cdot \boldsymbol{\xi}=0$ gives $\xi_{\phi} \equiv \boldsymbol{\xi} \cdot \hat{\mathbf{e}}_{\phi}=\left(R \xi_{R}\right)^{\prime} / i n$. Inserting into the radial component of Eq. (5) we obtain

$$
\left[P R\left(R \xi_{R}\right)^{\prime}\right]^{\prime}-Q \xi_{R}=0,
$$

where $P=\rho \omega_{D}^{2} / n^{2}$ and

$$
Q=\rho \omega_{D}^{2}+R\left(\frac{2 \rho \omega_{D} \Omega}{n}-\rho \Omega^{2}\right)^{\prime}+\rho^{\prime} \Phi^{\prime} .
$$

For $\omega_{D} \approx 0$, which is appropriate for marginally stable radially highly localized modes, this reduces to

$$
Q \approx \rho^{\prime}\left(\Phi^{\prime}-R \Omega^{2}\right)=-p^{\prime} \rho^{\prime} / \rho .
$$

This is the term responsible for the Rayleigh-Taylor instability, with the centrifugal acceleration added to the gravitational acceleration. We note the interesting cancellation of the MRI term $\rho \Omega^{2^{\prime}}$ due to the radial variation in the Doppler shifted frequency $\omega_{D}^{\prime}=n \Omega^{\prime}$. This cancellation can only occur for nonaxisymmetric modes with $n \neq 0$. How exactly does the canceling term arise?

Equation (7) shows that an incompressible radial perturbation $\xi_{R}$ is deflected in the $\hat{\mathbf{e}}_{\phi}$ direction by the Coriolis force or, especially when $\omega_{D} \approx 0$, gives rise to a pressure perturbation. Away from $\omega_{D}=0$ the Coriolis force increases, giving rise to a radially varying perturbed pressure. The resulting force, from Eq. (7),

$$
-\delta p^{\prime} \approx \frac{2 \rho \Omega}{n} \omega_{D}^{\prime} R \xi_{R}=\rho \Omega^{2^{\prime}} R \xi_{R}
$$

is restoring for a radially decreasing angular rotation frequency. This stabilizing effect exactly cancels the destabilizing effect of the MRI.

\section{B. Arbitrary vertical wavelength}

In the preceding section we assumed no variation of the mode with the axial coordinate $z$. Here we take $\xi \sim$ $e^{-i\left(n \phi+k_{z} z+\omega t\right)}$ and for generality include an axial velocity component $u_{z}$. Solving for $\xi_{z}, \xi_{\phi}$, and $\delta p$ from projections of Eq. (5) in the $\hat{\mathbf{e}}_{z}$ and $\hat{\mathbf{e}}_{\phi}$ directions and the incompressibility condition gives

$$
\begin{array}{r}
\xi_{\phi}=-i \frac{n\left(R \xi_{R}\right)^{\prime}+2 \frac{\Omega \omega_{D}}{\omega_{D}^{2}} k_{z}^{2} R^{2} \xi_{R}}{\bar{k}^{2} R^{2}}, \\
\delta p=\frac{\rho \omega_{D}^{2}\left(R \xi_{R}\right)^{\prime}-2 n \rho \omega_{D} \Omega \xi_{R}}{\bar{k}^{2} R},
\end{array}
$$

where $\bar{k}^{2}=n^{2} / R^{2}+k_{z}^{2}$ and $\omega_{D}=\omega+n \Omega+k_{z} u_{z}$. The vertical perturbation $\xi_{z}=-i k_{z} \delta p / \rho \omega_{D}^{2}$ is not needed here. Inserting Eqs. (12) and (13) into the radial component of Eq. (5) gives again Eq. (8) with now

$$
P=\frac{\rho \omega_{D}^{2}}{R^{2} \bar{k}^{2}}
$$

and

$$
Q=\rho \omega_{D}^{2}+R\left(\frac{2 n \rho \omega_{D} \Omega}{R^{2} \bar{k}^{2}}-\rho \Omega^{2}\right)^{\prime}+\rho^{\prime} \Phi^{\prime}+\rho h \frac{k_{z}^{2}}{\bar{k}^{2}} .
$$

The only difference between Eqs. (15) and (9) is the final term, where

$$
h=-\frac{\left(2 \omega_{D} \Omega\right)^{2}}{\omega_{D}^{2}}
$$

Equation (15) can be rewritten as

$$
Q=\rho \omega_{D}^{2}+2 n \omega_{D} R\left(\frac{\rho \Omega}{R^{2} \bar{k}^{2}}\right)^{\prime}+\rho^{\prime}\left(\Phi^{\prime}-R \Omega^{2}\right)-\rho \kappa^{2} \frac{k_{z}^{2}}{\bar{k}^{2}},
$$

where $\kappa^{2}=R \Omega^{2^{\prime}}+4 \Omega^{2}$ is the squared epicyclic frequency, associated with inertial circles. The pressure-Coriolis term proportional to $\omega_{D}^{\prime}$ now only partially cancels the centrifugal term $\rho \Omega^{2^{\prime}}$, resulting in the prefactor $k_{z}^{2} / \bar{k}^{2}$. The cancellation is therefore only complete for $k_{z}=0$.

\section{Including a magnetic field}

When imposing a vertical magnetic field, differential rotation will generate an azimuthal field as well. We therefore consider a general magnetic field $\mathbf{B}=B_{z}(R) \hat{\mathbf{e}}_{z}+B_{\phi}(R) \hat{\mathbf{e}}_{\phi}$. With $\nabla \times \mathbf{B} \times \mathbf{B}=-\frac{1}{2} \nabla B^{2}+\mathbf{B} \cdot \nabla \mathbf{B}$ the radial component of the force balance equation (4) becomes

$$
\left(p+\frac{B^{2}}{2}\right)^{\prime}=\rho R\left(\Omega^{2}-\omega_{A \phi}^{2}-\frac{\Phi^{\prime}}{R}\right),
$$

where $\omega_{A \phi}=B_{\phi} /(R \sqrt{\rho})$. Here $-\omega_{A \phi}^{2}$ derives from the magnetic field line bending term $\mathbf{B} \cdot \nabla \mathbf{B}$ in the same way that $\Omega^{2}$ derives from the inertial term $-\mathbf{u} \cdot \nabla \mathbf{u}$.

The structure of the mode equation (8) does not change upon the introduction of a magnetic field. Equations (12), (13) and the expressions for $P=\rho \omega_{D}^{2} / R^{2} \bar{k}^{2}$ and $Q$ of Eq. (15) are 
unchanged, except for the replacements

$$
\begin{gathered}
\omega_{D}^{2} \rightarrow \omega_{D}^{2}-\omega_{A}^{2}, \\
\omega_{D} \Omega \rightarrow \omega_{D} \Omega+\omega_{A} \omega_{A \phi}, \\
\Omega^{2} \rightarrow \Omega^{2}-\omega_{A \phi}^{2},
\end{gathered}
$$

where the Alfvén frequency reads

$$
\omega_{A}^{2}=\frac{n^{2} B_{\phi}^{2} / R^{2}+k_{z}^{2} B_{z}^{2}}{\rho} .
$$

The resulting mode equation was first derived in Ref. [40] under the Boussinesq approximation. See for example Ref. [28] for a derivation. How the resulting $P$ and $Q$ relate to stability is elaborated in Appendix B in which various stability criteria are derived.

We note that the replacements of Eqs. (19)-(21) should be made before the simplifications of Eq. (17) are made. This is because with these replacements Eq. (16) reads

$$
h=-4 \frac{\left(\omega_{D} \Omega+\omega_{A} \omega_{A \phi}\right)^{2}}{\omega_{D}^{2}-\omega_{A}^{2}}= \begin{cases}-4 \Omega^{2} & (B=0), \\ 4 \omega_{A \phi}^{2} & \left(B \neq 0, \omega_{D} \rightarrow 0\right) .\end{cases}
$$

The loss of the stabilizing Coriolis term $-4 \Omega^{2}$ upon the introduction of even a very small magnetic field can give rise to the MRI.

As we have seen, the Coriolis force induces a pressure perturbation that can exactly cancel the MRI term. A fraction $k_{z}^{2} / \bar{k}^{2}$ of the MRI drive remains so that long wavelengths will be less unstable than smaller wavelengths. Since this only holds for nonaxisymmetric modes, the axisymmetric MRI will typically be more unstable than its nonaxisymmetric counterpart.

Next we turn to a toroidal magnetically confined plasma, where we will see that things are very similar except that there the cancellation with the MRI term is incomplete.

\section{TOROIDAL ANALYSIS}

We now switch to a toroidal geometry and will take into account plasma compressibility. We exclude a gravitational field so that the equilibrium is given by Eq. (4) with $\Phi=0$. For the magnetic field we write

$$
\mathbf{B}=\nabla \phi \times \nabla \psi+F \nabla \phi,
$$

with $F(\psi)=R B_{\phi}$. The poloidal magnetic flux $\psi$ provides a label for the nested magnetic surfaces. Normal to these surfaces, pressure forces are balanced by Lorentz forces as described by the Grad-Shafranov equation

$$
R^{2} \nabla \cdot\left(\frac{\nabla \psi}{R^{2}}\right)=-F F^{\prime}-R^{2} \frac{\partial p}{\partial \psi} .
$$

Within the magnetic surfaces the force balance is given by $\partial p /\left.\partial R\right|_{\psi}=\rho R \Omega^{2}$ which can be solved analytically by [14]

$$
\begin{gathered}
p=p_{0}\left(1+\frac{R^{2}-R_{0}^{2}}{R_{0}^{2}} \frac{\mathcal{M}^{2}}{\zeta}\right)^{\zeta}, \\
\rho=\rho_{0}\left(1+\frac{R^{2}-R_{0}^{2}}{R_{0}^{2}} \frac{\mathcal{M}^{2}}{\zeta}\right)^{\zeta-1},
\end{gathered}
$$

where $p_{0}, \rho_{0}$, and $\zeta$ are arbitrary nonzero functions of only $\psi$. The quantity $\mathcal{M} \equiv R_{0} \Omega \sqrt{\rho_{0} / 2 p_{0}}$ is approximately a Mach number. The quantity $\gamma_{\zeta} \equiv \zeta /(\zeta-1)$ can be interpreted as the adiabatic constant of the equilibrium [14].

We perform an expansion in terms of the inverse aspect ratio $\epsilon=r / R_{0}$. We consider only the leading order terms in $\epsilon$, so that the Shafranov shift does not enter and we can use simple polar coordinates $(r, \theta)$ in the poloidal plane. The approach we will follow is similar to that of Ref. [3]. We will however allow for an arbitrary value of the adiabatic constant of both the dynamics $\gamma$ and the equilibrium $\gamma_{\zeta}$, both of which are taken equal to one in [3]. For $\epsilon \ll 1$, poloidal Fourier harmonics $\propto e^{i m \theta}$ couple weakly so that we can write

$$
\xi_{r}=\sum_{m^{\prime}=m, m \pm 1} \xi_{r}^{m^{\prime}} e^{i\left(m^{\prime} \theta-n \phi-\omega t\right)},
$$

with $\xi_{r}^{m \pm 1} \sim \epsilon \xi_{r}^{m}$ and similarly for the other components $\xi_{\theta}, \xi_{\phi}$. We order $\beta \equiv 2 p_{0} / B_{0}^{2} \sim \epsilon^{2}$ and $\omega_{D} \sim \Omega \sim \epsilon \sqrt{p_{0} / \rho_{0}}$ so that $\mathcal{M} \sim 1$ as in Refs. [3,4,6]. A consistent ordering turns out to require for the component parallel to the magnetic field $\xi_{\|}^{m} \sim \epsilon \xi_{r}^{m}$, but $\xi_{\|}^{m \pm 1} \sim \xi_{r}^{m} \sim \xi_{\theta}^{m}$. The components $\xi_{R}, \xi_{z}$, and $\xi_{\phi}$ are ordered the same as $\xi_{\|}$.

Making use of the equilibrium relation (4), the projection of Eq. (5) in the direction of $\mathbf{B}$ can be written as [5]

$$
\begin{aligned}
- & \rho \omega_{D}^{2}(\mathbf{B} \cdot \boldsymbol{\xi})+2 i \rho \omega_{D} \Omega\left(\mathbf{B} \times \hat{\mathbf{e}}_{z}\right) \cdot \boldsymbol{\xi} \\
& =\mathbf{B} \cdot \nabla(\gamma p \nabla \cdot \boldsymbol{\xi})+\rho \Omega^{2} \mathbf{B} \cdot[\nabla(\boldsymbol{\xi} \cdot \mathbf{R})-\mathbf{R} \nabla \cdot \boldsymbol{\xi}] .
\end{aligned}
$$

To leading order in $\epsilon$ this becomes

$$
-\rho_{0} \omega_{D}^{2} \xi_{\|}-2 i \rho_{0} \omega_{D} \Omega \xi_{R}=i k_{\|}\left(\gamma p_{0} \nabla \cdot \xi+\rho_{0} \Omega^{2} R \xi_{R}\right),
$$

where acting on an $m^{\prime}$ Fourier harmonic

$$
k_{\|}^{m^{\prime}} \equiv \frac{\mathbf{B}}{B} \cdot \nabla \approx \frac{1}{R_{0}}\left(\frac{m^{\prime}}{q}-n\right) \sim \epsilon,
$$

with the safety factor $q=r B_{\phi} / R_{0} B_{\theta}+O(\epsilon)$.

To solve for $\xi_{\|}$from Eq. (30) we need $\nabla \cdot \xi$. For a low $\beta \sim \epsilon^{2}$ plasma, the toroidal magnetic field perturbation $\delta F=$ $-R^{2} \nabla \cdot\left(F \xi_{p} / R^{2}\right)$, with $\boldsymbol{\xi}_{p}=\boldsymbol{\xi}-\xi_{\phi} \hat{\mathbf{e}}_{\phi}$, creates such a large restoring force that for instabilities it has to vanish to order $\epsilon^{2}[15,16]$. Therefore

$$
\nabla \cdot \boldsymbol{\xi}_{p} \approx \boldsymbol{\xi}_{p} \cdot\left(\frac{2 \nabla R}{R}-\frac{\nabla F}{F}\right)=\frac{2 \xi_{R}}{R}+O\left(\epsilon^{2}\right),
$$

where we used that Eq. (25) gives $F^{\prime} / F \sim \beta^{\prime} \sim \epsilon^{2}$. With Eq. (31) we thus obtain to leading order

$$
\nabla \cdot \xi=\frac{2 \xi_{R}}{R}+i k_{\|} \xi_{\|} \sim \epsilon .
$$

Equation (30) now gives

$$
\begin{aligned}
\xi_{\|}^{m \pm 1} & =\frac{2 i \rho_{0} \omega_{D} \Omega+i k_{\|}^{m \pm 1}\left(\frac{2 \gamma p_{0}}{R_{0}}+\rho_{0} R_{0} \Omega^{2}\right)}{\gamma p_{0}\left(k_{\|}^{m \pm 1}\right)^{2}-\rho_{0} \omega_{D}^{2}} \xi_{R}^{m \pm 1}, \\
& \approx 2 i \frac{\omega_{D} \Omega \pm q \omega_{0}^{2}\left(1+\frac{\mathcal{M}^{2}}{\gamma}\right)}{\omega_{0}^{2}-\omega_{D}^{2}} \xi_{R}^{m \pm 1}
\end{aligned}
$$

with $\omega_{0}=\sqrt{\gamma p_{0} / \rho_{0}} / q R_{0}$. The approximation in Eq. (34) holds when we order $k_{\|}^{m} \sim \epsilon^{2}$ so that $k_{\|}^{m \pm 1} \approx \pm 1 / q R_{0} \sim \epsilon$. By ordering $k_{\|}^{m}$ an order lower than implied by Eq. (31), we 
focus on a region close to a rational surface where $q=m / n$. From Eq. (32) we have to zeroth order in $\epsilon$ that $\nabla \cdot \xi_{p}=0$ so that $\xi_{\theta}^{m}=i\left(r \xi_{r}^{m}\right)^{\prime} / m$. Therefore, to leading order we find for $\xi_{R}=\cos \theta \xi_{r}-\sin \theta \xi_{\theta}$

$$
\xi_{R}^{m \pm 1}=\frac{1}{2}\left(\xi_{r}^{m} \mp \frac{\left(r \xi_{r}^{m}\right)^{\prime}}{m}\right) .
$$

In the following we will not take into account the effect of the magnetic field perturbations explicitly, so that $\mathbf{F}_{s}=-\nabla \delta p$. We take the divergence of the cross-product of Eq. (5) with $\tilde{\mathbf{B}} \equiv \mathbf{B} / B^{2}$, to obtain after various vector operations

$$
\begin{aligned}
0= & \hat{\mathbf{e}}_{R} \times \tilde{\mathbf{B}} \cdot \nabla \rho \omega_{D}^{2} \xi_{R}+\hat{\mathbf{e}}_{z} \times \tilde{\mathbf{B}} \cdot \nabla \rho \omega_{D}^{2} \xi_{z}+2 i \hat{\mathbf{e}}_{z} \cdot \nabla \rho \omega_{D} \Omega \frac{\xi_{\|}}{B}+\frac{2 \tilde{\mathbf{B}} \times \nabla B}{B} \cdot \nabla \delta p+\frac{\tilde{\mathbf{B}} \times \hat{\mathbf{e}}_{R}}{R} \cdot \nabla\left(R^{2} \nabla \cdot\left(\rho \Omega^{2} \boldsymbol{\xi}\right)\right) \\
& +\rho \omega_{D}^{2}\left(\xi_{R} \nabla \cdot \hat{\mathbf{e}}_{R} \times \tilde{\mathbf{B}}+\xi_{z} \nabla \cdot \hat{\mathbf{e}}_{z} \times \tilde{\mathbf{B}}\right)-2 i \nabla \cdot\left(\rho \omega_{D} \Omega \tilde{\mathbf{B}} \cdot \hat{\mathbf{e}}_{z} \boldsymbol{\xi}\right)+\frac{\nabla \times \mathbf{B}}{B^{2}} \cdot \nabla \delta p+R^{2} \nabla \cdot\left(\rho \Omega^{2} \boldsymbol{\xi}\right) \nabla \cdot\left(\frac{\tilde{\mathbf{B}} \times \hat{\mathbf{e}}_{R}}{R}\right) .
\end{aligned}
$$

We can write, using the covariant component $\xi_{\psi} \equiv \xi \cdot \partial \mathbf{r} / \partial \psi$

$$
\begin{gathered}
\nabla \cdot \rho \Omega^{2} \boldsymbol{\xi}=\rho \Omega^{2} \nabla \cdot \boldsymbol{\xi}+\frac{R\left(\rho \Omega^{2}\right)^{2}}{\gamma_{\zeta} p} \xi_{R}+\frac{\partial \rho \Omega^{2}}{\partial \psi} \xi_{\psi}, \\
\delta p=-\gamma p \nabla \cdot \boldsymbol{\xi}-\rho R \Omega^{2} \xi_{R}-\frac{\partial p}{\partial \psi} \xi_{\psi} .
\end{gathered}
$$

The final terms of Eq. (37) and (38) give rise to the centrifugal and pressure terms of Eq. (1). We will not take them into account here. To leading order, Eq. (36) then greatly simplifies. The entire second line vanishes. We can approximate $\hat{\mathbf{e}}_{R} \times$ $\tilde{\mathbf{B}} \approx-\hat{\mathbf{e}}_{z} / B$, and $\hat{\mathbf{e}}_{z} \times \tilde{\mathbf{B}}=\hat{\mathbf{e}}_{R} / B$ so that Eq. (36) can be written as $(2 / B) \partial f / \partial z=0$ where

$$
\begin{aligned}
f= & -\rho_{0} \omega_{D}^{2} \xi_{R}+i \rho_{0} \omega_{D} \Omega \xi_{\|}+\frac{\gamma p_{0}}{R_{0}^{2}}\left(1+\frac{\mathcal{M}^{2}}{\gamma}\right) R_{0} \nabla \cdot \xi \\
& +\rho_{0} \Omega^{2}\left(1+\frac{\mathcal{M}^{2}}{\gamma_{\zeta}}\right) \xi_{R} .
\end{aligned}
$$

To rewrite the second term of Eq. (36) we used that $\boldsymbol{\nabla} \times \boldsymbol{\xi}_{p}=$ 0 to zeroth order in $\epsilon$, so that $\partial \xi_{z} / \partial R=-\partial \xi_{R} / \partial z$. Using Eqs. (33) and (34) the poloidal harmonics of $f$ can be written as $f^{m \pm 1}=(a \pm b) \xi_{R}^{m \pm 1}$, where

$$
\begin{aligned}
& a=\frac{\rho_{0}\left(\omega_{D}^{2}-\omega_{-}^{2}\right)\left(\omega_{D}^{2}-\omega_{+}^{2}\right)}{\omega_{D}^{2}-\omega_{0}^{2}}, \\
& b=\frac{4 q \rho_{0} \omega_{D} \Omega \omega_{0}^{2}}{\omega_{0}^{2}-\omega_{D}^{2}}\left(1+\frac{\mathcal{M}^{2}}{\gamma}\right) .
\end{aligned}
$$

Here $\omega_{ \pm}^{2}=\left(\omega_{0}^{2} / 2\right)\left(a_{1} \pm \sqrt{a_{1}^{2}-a_{2}}\right)$, with

$$
\begin{aligned}
& a_{1}=1+2 q^{2}\left(1+\frac{4 \mathcal{M}^{2}}{\gamma}+\frac{\mathcal{M}^{4}}{\gamma \gamma_{\zeta}}\right), \\
& a_{2}=\frac{2 q^{2} \mathcal{M}^{4}}{\gamma}\left(\frac{1}{\gamma_{\zeta}}-\frac{1}{\gamma}\right),
\end{aligned}
$$

the zonal flow and geodesic acoustic mode frequencies, respectively. These frequencies were first derived for $\gamma_{\zeta}=1$ in Refs. [17,18], for $\gamma_{\zeta} \rightarrow \infty$ in Ref. [19], and for general $\gamma_{\zeta}$ in Ref. [14]. To leading order, $\partial / \partial z=\sin \theta \partial / \partial r+\cos \theta \partial / r \partial \theta$ so that

$$
\begin{aligned}
2 i \frac{\partial f}{\partial z} & =\left(\frac{\partial}{\partial r}-\frac{m-1}{r}\right) f^{m-1}-\left(\frac{\partial}{\partial r}+\frac{m+1}{r}\right) f^{m+1} \\
& =\frac{1}{r}\left[r\left(f^{m-1}-f^{m+1}\right)\right]^{\prime}-\frac{m}{r}\left(f^{m-1}+f^{m+1}\right) .
\end{aligned}
$$

With $2 f^{m \pm 1}=(a \pm b)\left[\xi_{r} \mp\left(r \xi_{r}\right)^{\prime} / m\right]$, we obtain

$$
\begin{aligned}
2 i m r \frac{\partial f}{\partial z} & =\left\{\left[\operatorname{ar}\left(r \xi_{r}\right)^{\prime}-b m r \xi_{r}\right]\right\}^{\prime}-\left[a m^{2} \xi_{r}-b m\left(r \xi_{r}\right)^{\prime}\right] \\
& =\left[\operatorname{ar}\left(r \xi_{r}\right)^{\prime}\right]^{\prime}-m^{2}\left[a+r(b / m)^{\prime}\right] \xi_{r}=0,
\end{aligned}
$$

which is equal in structure to the cylindrical mode equation (8). The magnetorotational effect, from the final term of Eq. (37) that we did not take into account, effectively adds $\rho_{0} \Omega^{2}(1+$ $\mathcal{M}^{2}$ ) to $b / m[3,4]$. For $\mathcal{M} \ll 1$ this gives the second and third term on the right-hand side of Eq. (2). With Eq. (41), $(b / m)^{\prime}$ gives only the Coriolis-pressure contribution. It derives from two equal contributions of the Coriolis and perturbed pressure terms, the second and third terms of Eq. (39), respectively. For $\omega_{D} \approx 0$ and $\mathcal{M}^{2} \ll 1$, Eq. (41) gives

$$
\left(\frac{b}{m}\right)^{\prime} \approx \frac{4 \rho_{0} \omega_{D}^{\prime} \Omega}{n}=2 \rho_{0} \Omega^{2^{\prime}},
$$

which is the first term on the right-hand side of Eq. (2). Comparing this with the corresponding term in the cylindrical mode equation (8), we find that it is twice as large in a toroidal geometry. Consequently, only half of its effect is canceled by the magnetorotational effect. This allows flow shear to be stabilizing in a toroidal geometry.

\section{SIMULATIONS}

Next, we perform numerical computations using FINESSE [20] and PHOENIX [21,22]. We take a circular equilibrium with $\epsilon=0.3$ and use profiles $\beta_{0} \approx 0.01\left(1-\psi_{n}\right)^{2}$ and $\rho_{0} \propto 1-\psi_{n}$, where $\psi_{n}$ is the poloidal flux scaled to zero on the magnetic axis and unity at the plasma edge. The profile $F F^{\prime} \propto-0.95 \psi_{n}-$ $0.1 \psi_{n}+3 \psi_{n}^{5}$ gives $q \approx 1$ in the core of the plasma while increasing steeply near the edge to a value above 3.5. This is relevant for the "hybrid scenario" envisioned for advanced operation of the ITER tokamak. In the entire core of the plasma, 


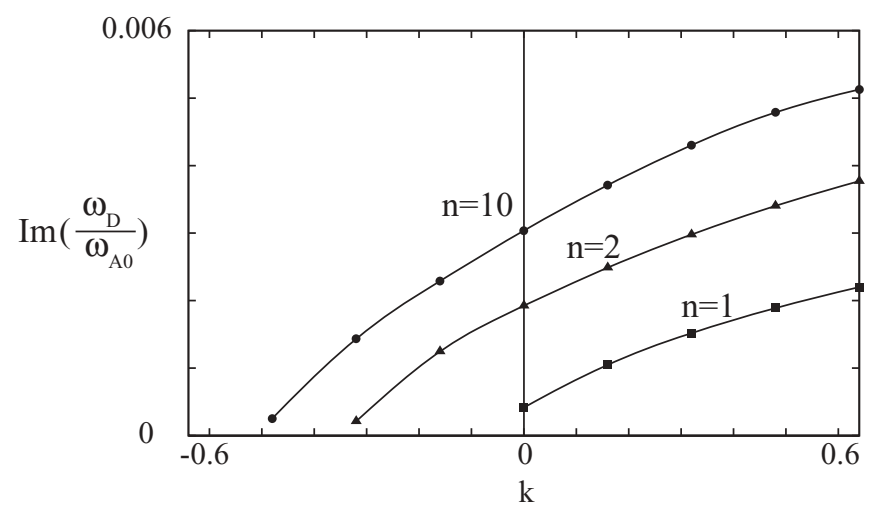

FIG. 1. The normalized growth rate as a function of the flow shear $k=d \ln \Omega / d \psi_{n}$ at $\psi_{n}=0.1$ for various toroidal mode numbers $n$.

starting from $q=0.995$ on axis, the magnetic shear is very low, with $q^{\prime}=0.03$ at $\psi_{n}=0.1$ where $q=1$.

With $q \approx 1$ in the center, the stability criterion of Eq. (1) for rigid rotation reads

$$
\frac{r}{4}\left(\frac{q^{\prime}}{q^{2}}\right)^{2}>\frac{\rho_{0}^{\prime} \Omega^{2}}{\rho_{0} \omega_{A}^{2}},
$$

where $\omega_{A}=B_{0} / R_{0} \sqrt{\rho_{0}}$. The destabilizing centrifugal term on the right-hand side results in modes similar to those of Ref. [7] with growth rates of several tens of a percent of $\omega_{A 0}$ on the magnetic axis. We use a rotation profile

$$
\Omega / \omega_{A 0} \approx 0.02\left[1+k\left(\psi_{n}-0.1\right)\right],
$$

giving $\mathcal{M}=\sqrt{\beta} \Omega / \omega_{A} \approx 0.2$ at $\psi_{n}=0.1$.

Near the plasma edge, a resistive computation with very high radial resolution is required to resolve the eigenfunctions. The growth rates were however found to be quite insensitive to applying ideally conducting wall boundary conditions at $\sqrt{\psi_{n}}=0.8$, where $q \approx 1.1$. A resolution of 100 radial elements and seven poloidal Fourier harmonics was in this case found to be sufficient.

Figure 1 shows the growth rate of the most unstable modes as a function of the flow shear. It shows stabilization for radially decreasing rotation $k<0$ and destabilization for radially increasing rotation $k>0$, in correspondence with the theoretical analysis of this work. The second derivative of the growth rate with respect to $k$ is negative. This may very well be due to the shearing effect investigated for ballooning modes in Refs. [23,24], which is always stabilizing. Indeed, for a similar equilibrium with a higher value of $\beta \approx 0.05$ on axis, the growth rate of the higher $n$ modes was found to decrease with increasing positive flow shear $k$.

\section{DISCUSSION}

We showed that a stabilizing effect of a radially decreasing angular rotation frequency on nonaxisymmetric modes originates from the Coriolis effect. In spite of being perpendicular to the displacement, the Coriolis effect has a significant impact on stability through the mediating role of the perturbed pressure. In the uncommon case that the angular rotation frequency increases with minor radius, the effect can even be destabilizing. In a rotating cylindrical plasma the effect on nonaxisymmetric modes with no vertical variation is exactly canceled by the centrifugal effect responsible for the MRI. The effect may therefore be important for the recently observed nonaxisymmetric MRI [25]. We expect modes with a long vertical wavelength to be highly suppressed. Since the effect results from a radial variation in the Doppler shifted mode frequency, it does not influence axisymmetric modes like the classical MRI.

The effect has often been missed in the literature. In Refs. [3,4] $\omega_{D}^{\prime}$ was incorrectly neglected along with $\omega_{D}$. In Ref. [26] and later again in [27] an error was made in the WKB analysis as explained in [28]. In Ref. [29] the perturbed pressure $\delta p$ was not included so that the effect was missed. In a cylindrical geometry the effect $i s$ included in the original analysis of [30] or more recently in for example [31,32]. The fact that without axial variation the Coriolis force only modifies the pressure was already observed in Ref. [33].

Instability resulting from the Coriolis-pressure effect may be interpreted as a co-rotation instability. The part of the mode inside the co-rotation radius where $\omega_{D}=0$ moves slower than the plasma and has negative energy [34,35]. Energy transfer to the positive energy part of the mode outside the co-rotation radius can cause growth of the mode. See Appendix C.

Relative to pressure effects, the effect discussed in this work scales with $\mathcal{M}^{2}$, so it will become particularly significant for high Mach number plasmas. In the plasma core of hybrid scenario plasmas, with a safety factor close to one, plasma pressure effects are small. Toroidal flow shear can therefore aid the formation of internal transport barriers, by stabilizing magnetohydrodynamic instabilities. Because of its dependence on the sign of the flow shear, the pressure-Coriolis effect can be discriminated from other effects like that of Ref. [23,24], the parallel velocity gradient or Kelvin-Helmholtz instability, and nonlinear flow shear suppression.

Also for stable modes, the Coriolis-pressure effect can be relevant. From Eq. (41), this particularly holds for modes with a frequency $\omega_{D}^{2} \approx \omega_{0}^{2}$, although such a singularity is typically something that is "smoothed" in a more complete kinetic analysis. In Ref. [36], the Coriolis-pressure effect was predicted to influence the existence of reversed shear Alfvén eigenmodes with $\omega_{D}^{2} \approx \omega_{+}^{2}$, favoring modes propagating in the direction of the plasma rotation [36].

\section{ACKNOWLEDGMENTS}

We thank J. P. Goedbloed, E. Westerhof, and B. Koren for comments. This work, supported by NWO and the European Communities under the contract of the Association EURATOM/FOM, was carried out within the framework of the European Fusion Program.

\section{APPENDIX A: FRIEMAN-ROTENBERG EQUATION}

\section{General}

We adopt a Lagrangian viewpoint in which we move along with the flow. A Lagrangian perturbation of a quantity $f$ with equilibrium value $f_{0}$ will be defined as $\delta_{L} f \equiv f(\mathbf{r}+\boldsymbol{\xi})-$ $f_{0}(\mathbf{r})$ [37], related to the Eulerian perturbation $\delta_{E}=\delta_{L}-\boldsymbol{\xi}$. $\nabla$. Here the so-called Lagrangian displacement vector $\boldsymbol{\xi}(\mathbf{r}, t)=$ 
$\delta_{L} \mathbf{r}$ gives the displacement of a fluid element that would have been at position $\mathbf{r}$ at time $t$ in the unperturbed flow.

We introduce the advective derivative $D / D t=\partial / \partial t+\mathbf{u}$. $\nabla$ which commutes with $\delta_{L}$ [37]. It then follows from $\mathbf{u}=$ $D \mathbf{r} / D t$ that $\delta_{L} \mathbf{u}=D \xi / D t$. The Frieman-Rotenberg equation is obtained by acting with $\delta_{L}$ on the momentum equation $\rho D \mathbf{u} / D t=\mathbf{f}$, giving

$$
\left(\delta_{L} \rho\right) \frac{D \mathbf{u}}{D t}+\rho \frac{D^{2} \boldsymbol{\xi}}{D t^{2}}=\delta_{L} \mathbf{f} .
$$

Similarly, acting with $\delta_{L}$ on the continuity equation $D \rho / D t=$ $-\rho \boldsymbol{\nabla} \cdot \mathbf{u}$ gives $\delta_{L} \rho=-\rho \boldsymbol{\nabla} \cdot \boldsymbol{\xi}$ so that the first term of Eq. (A1) can be written as

$$
-\rho(\nabla \cdot \xi) \frac{D \mathbf{u}}{D t}=-\nabla \cdot\left(\xi \rho \frac{D \mathbf{u}}{D t}\right)+\xi \cdot \nabla\left(\rho \frac{D \mathbf{u}}{D t}\right) .
$$

We define the static force operator $\mathbf{F}_{s}(\boldsymbol{\xi}) \equiv \delta_{E} \mathbf{f}$, where in the rest of the text we suppress the argument $\boldsymbol{\xi}$. Equation (A1) now gives the Frieman-Rotenberg equation [12]

$$
\rho\left(\frac{\partial}{\partial t}+\mathbf{u} \cdot \nabla\right)^{2} \boldsymbol{\xi}=\mathbf{F}_{s}+\nabla \cdot(\boldsymbol{\xi} \rho \mathbf{u} \cdot \nabla \mathbf{u}),
$$

where we used that in equilibrium $D \mathbf{u} / D t=\mathbf{u} \cdot \nabla \mathbf{u}$. We remark that the right-hand side of Eq. (A3) actually represents the self-adjoint Galilean invariant generalized force operator deployed for example in Refs. [38,39]. The total force operator of Frieman and Rotenberg [12] is given by

$$
\mathbf{F}=\mathbf{F}_{s}+\nabla \cdot(\boldsymbol{\xi} \rho \mathbf{u} \cdot \nabla \mathbf{u})-\rho(\mathbf{u} \cdot \nabla)^{2} \boldsymbol{\xi},
$$

so that Eq. (A3) can be written as

$$
\rho \frac{\partial^{2} \boldsymbol{\xi}}{\partial t^{2}}+2 \rho \mathbf{u} \cdot \nabla \frac{\partial \boldsymbol{\xi}}{\partial t}=\mathbf{F}
$$

\section{Normal modes}

Considering normal modes $\xi \sim e^{-i\left(\omega t+n \phi+k_{z} z\right)}$ and a velocity $\mathbf{u}=R \Omega \hat{\mathbf{e}}_{\phi}+u_{z} \hat{\mathbf{e}}_{z}$ we have with $\omega_{D}=\omega+n \Omega+k_{z} u_{z}$ and $\boldsymbol{\Omega}=-\boldsymbol{\Omega} \hat{\mathbf{e}}_{z}$

$$
\frac{D \xi}{D t}=-i \omega_{D} \xi+\Omega \times \xi,
$$

so that

$$
\frac{D^{2} \boldsymbol{\xi}}{D t^{2}}=-\omega_{D}^{2} \xi-i \omega_{D} \boldsymbol{\Omega} \times \boldsymbol{\xi}+\frac{D}{D t}(\boldsymbol{\Omega} \times \boldsymbol{\xi}) .
$$

The final term reads

$$
\boldsymbol{\Omega} \times \frac{D \boldsymbol{\xi}}{D t}=-i \omega_{D} \boldsymbol{\Omega} \times \boldsymbol{\xi}+\boldsymbol{\Omega} \times \boldsymbol{\Omega} \times \boldsymbol{\xi} .
$$

The first term of Eq. (A8) combines with the second term of Eq. (A7) to give the Coriolis force. For the right-hand side of Eq. (A3) we need

$$
\begin{aligned}
\nabla \cdot(\boldsymbol{\xi} \rho \mathbf{u} \cdot \nabla \mathbf{u}) & =-\nabla \cdot\left(\boldsymbol{\xi} \rho \Omega^{2} \mathbf{R}\right) \\
& =-\mathbf{R} \cdot \nabla\left(\rho \Omega^{2} \boldsymbol{\xi}\right)-\rho \Omega^{2} \boldsymbol{\xi} \cdot \nabla \mathbf{R} .
\end{aligned}
$$

The last term can be written $-\Omega^{2}\left(\xi_{R} \hat{\mathbf{e}}_{R}+\xi_{\phi} \hat{\mathbf{e}}_{\phi}\right)$ and exactly cancels the last term of Eq. (A8). The Frieman-Rotenberg equation (A3) can then be written as Eq. (5).

\section{APPENDIX B: STABILITY ANALYSIS}

In this section we will investigate stability. First we show a very general criterion that determines stability a posteriori. Next we investigate how the coefficients $P$ and $Q$ of the mode equation (8) influence stability.

\section{A general criterion}

In the following we assume normal mode solutions $\boldsymbol{\xi} \sim$ $e^{-i \omega t}$. Taking the inner product of Eq. (A5) with the complex conjugate $\xi^{*}$ and integrating over the plasma volume we can solve the resulting quadratic equation to give solutions $\omega=\omega_{-}$ and $\omega_{+}$, where [12]

$$
\omega_{ \pm}=\bar{V} \pm \sqrt{\bar{V}^{2}+\bar{W}} .
$$

A bar denotes normalization with $I=\frac{1}{2} \int \rho|\xi|^{2} d^{3} \mathbf{r}$ and

$$
\begin{aligned}
V & =\frac{1}{2} \int \xi^{*} \cdot(-i \rho \mathbf{u} \cdot \nabla \boldsymbol{\xi}) d^{3} \mathbf{r} \\
W & =\frac{1}{2} \int-\xi^{*} \cdot \mathbf{F} d^{3} \mathbf{r}
\end{aligned}
$$

are both real [28]. Here $\bar{V}$ and $W$ are the average DopplerCoriolis shift and potential energy, respectively. The frequencies of Eq. (B1) are real when $\bar{W} \geqslant-\bar{V}^{2}$, providing a necessary and sufficient criterion for stability.

\section{WKB analysis}

We assume the radial variation of $\boldsymbol{\xi}$ is much faster than the length scale $L$ over which the equilibrium quantities vary. In this case we can insert WKB solutions $R \xi_{R} \sim e^{i \int k_{R} d R}$ into Eq. (8) and neglect terms higher order in $1 / L$ to obtain

$$
-k_{R}^{2} R^{2} P-Q=0 .
$$

We assume that either the magnetic field or $k_{z}$ vanishes so that the term $\rho h k_{z}^{2} / \bar{k}^{2}$ does not depend on the mode frequency. Then Eq. (B4) becomes a quadratic equation in $\omega_{D}$ with solution

$$
\omega_{D}=\overline{\mathcal{V}} \pm \sqrt{\overline{\mathcal{V}}^{2}+\overline{\mathcal{W}}}
$$

where with $k^{2}=k_{R}^{2}+\bar{k}^{2}$,

$$
\overline{\mathcal{V}}=-\frac{\bar{k}^{2}}{k^{2}} \frac{R}{\rho}\left(\frac{n \rho \Omega}{\bar{k}^{2} R^{2}}\right)^{\prime}
$$

and

$$
\begin{aligned}
\overline{\mathcal{W}}= & \omega_{A}^{2}-\frac{k_{z}^{2}}{k^{2}}\left(h-R \Omega^{2^{\prime}}\right)-2 \frac{n k_{z}}{R k^{2}} u_{z}^{\prime} \Omega \\
& -\frac{\bar{k}^{2}}{k^{2}} \frac{R}{\rho}\left[\rho^{\prime}\left(\frac{\Phi^{\prime}}{R}-\Omega^{2}\right)+\left(\rho \omega_{A \phi}^{2}\right)^{\prime}+\left(\frac{2 n \rho \omega_{A} \omega_{A \phi}}{\bar{k}^{2} R^{2}}\right)\right] .
\end{aligned}
$$

The equilibrium is stable against the rapidly varying $\mathrm{WKB}$ modes when $\overline{\mathcal{W}}>-\overline{\mathcal{V}}^{2}$.

Equation (B7) consists of a stabilizing Alfvén contribution and several potentially destabilizing terms. The first of these is the inertial term that becomes equal to $\left(k_{z}^{2} / k^{2}\right) \kappa^{2}$ in the absence of a magnetic field. The final term on the first line of 
Eq. (B7) was already found in Ref. [30] and arises through the radial variation of the Doppler shift.

On the second line of Eq. (B7) we have first the gravitational convective term augmented by the centrifugal force. Together with the next term, it may be rewritten using the equilibrium relation (18) as $\rho^{\prime}$ times the total pressure gradient. The remaining term proportional to $\left(\omega_{A \phi}^{2}\right)^{\prime}$ was in Ref. [43] argued to be responsible for the helical MRI [44,45]. This term is the magnetic field line bending analog of the centrifugal MRI term proportional to $\Omega^{2^{\prime}}$. The final term of Eq. (B7) is then analogous to the pressure-Coriolis term that canceled part of the MRI term to give the prefactor $k_{z}^{2} / k^{2}$. Because of the sign difference in Eqs. (20) and (21), this term however always adds to the previous. For example, for $k_{z}=0$ it reads $\left(2 \rho \omega_{A \phi}^{2}\right)^{\prime}$.

Comparing Eq. (B1) with (B5) we see that $\bar{V}=\bar{V}-n \Omega-$ $k_{z} u_{z}$. We would like to show, at least for $k_{z}=0$, that our result for $\overline{\mathcal{V}}$ of Eq. (B6) agrees with the definition of $\bar{V}$ given in Eq. (B2). We first write

$$
\begin{aligned}
\xi^{*} \cdot(-i \mathbf{u} \cdot \nabla \boldsymbol{\xi}) & =\xi^{*} \cdot\left(-n \Omega \xi+i \Omega \hat{\mathbf{e}}_{z} \times \xi\right) \\
& =-n \Omega|\xi|^{2}+i \Omega \hat{\mathbf{e}}_{z} \cdot\left(\xi \times \xi^{*}\right),
\end{aligned}
$$

where in $(R, z, \phi)$ coordinates

$$
i \hat{\mathbf{e}}_{z} \cdot\left(\boldsymbol{\xi} \times \boldsymbol{\xi}^{*}\right)=i\left(\xi_{\phi} \xi_{R}^{*}-\xi_{R} \xi_{\phi}^{*}\right)=\frac{\left(\left|R \xi_{R}\right|^{2}\right)^{\prime}}{n R} .
$$

In the final step we used that incompressibility implies $\xi_{\phi}=$ $\left(R \xi_{R}\right)^{\prime} / i n$. Therefore Eq. (B2) can be written as

$$
\begin{aligned}
\bar{V} & =-\frac{\int \rho \Omega\left(n|\boldsymbol{\xi}|^{2}-\left(\left|R \xi_{R}\right|^{2}\right)^{\prime} / n R\right) d^{3} \mathbf{r}}{\int \rho|\boldsymbol{\xi}|^{2} d^{3} \mathbf{r}}, \\
& =-\frac{\int\left[\rho n \Omega|\xi|^{2}+(\rho \Omega)^{\prime}\left|R \xi_{R}\right|^{2} / n R\right] d^{3} \mathbf{r}}{\int \rho|\boldsymbol{\xi}|^{2} d^{3} \mathbf{r}}, \\
& \approx-n \Omega-\frac{n}{\rho R k^{2}}(\rho \Omega)^{\prime} .
\end{aligned}
$$

On the second line we partially integrated using $d^{3} \mathbf{r}=$ $R d R d \phi d z$ assuming that at $R=0$ and the plasma edge $\left|R \xi_{R}\right|$ vanishes. Since we assume that the perturbation varies over a much smaller length scale, on the final line we took the equilibrium quantities out of the integrals. For evaluation of the remaining integrals we used that $\xi_{\phi}=k_{R} R \xi_{R} / n$. We thus find that indeed the result of Eq. (B13) agrees with Eq. (B6).

\section{Cluster point analysis}

Our mode equation (8) becomes singular for $P=0\left(\omega_{D}=\right.$ $\left.\omega_{A}\right)$, signaling the presence of a continuous spectrum. When also $P^{\prime}=0\left(\omega_{D}^{\prime}=\omega_{A}^{\prime}\right)$ a so-called cluster point may exist, where global modes cluster towards the continuum [46]. To leading order in the distance $s$ to this point, we can write Eq. (8) as $\left[s^{2}\left(R \xi_{R}\right)^{\prime}\right]^{\prime}+D R \xi_{R}=0$. Here

$$
D \equiv-\frac{Q}{\frac{1}{2} R(R P)^{\prime \prime}}
$$

is evaluated at $s=0$. This equation has solutions of the form $R \xi_{R}=s^{v}$ with $v=-1 / 2 \pm \sqrt{1 / 4-D}$. More formally we can perform a Frobenius expansion around $s=0$. Either way, we find that oscillatory behavior of the solutions cannot occur when $D<1 / 4$ providing a sufficient criterion for local stability. Using $\omega_{D}^{\prime}=n \Omega^{\prime}+k_{z} u_{z}^{\prime}$ this gives

$$
\begin{aligned}
D= & -\frac{\rho_{\bar{k}^{2}}^{2}\left(h-R \Omega^{2^{\prime}}\right)+\rho^{\prime}\left(\Phi^{\prime}-R \Omega^{2}\right)+\frac{2 n \rho k_{z} u_{z}^{\prime} \Omega}{R \bar{k}^{2}}}{\frac{1}{2} \rho\left(\omega_{A}^{2}-\omega_{D}^{2}\right)^{\prime \prime} R^{2}} \\
& -\frac{\omega_{D}\left(\frac{2 n \rho \Omega}{R^{2} \bar{k}^{2}}\right)^{\prime}+\left(\rho \omega_{A \phi}^{2}+\frac{2 n \rho \omega_{A} \omega_{A \phi}}{R^{2} \bar{k}^{2}}\right)^{\prime}}{\frac{1}{2} \rho\left(\omega_{A}^{2}-\omega_{D}^{2}\right)^{\prime \prime} R}<\frac{1}{4} .
\end{aligned}
$$

For highly localized modes, the Coriolis shift vanishes [28] so that at marginal stability $\omega_{D}=\omega_{A}=0$ and $\frac{1}{2}\left(\omega_{A}^{2}-\omega_{D}^{2}\right)^{\prime \prime}=$ $\omega_{A}^{\prime 2}-\omega_{D}^{\prime 2}$. When positive, both sides of Eq. (B15) can be multiplied with this quantity. The term $\omega_{A}^{\prime 2}$ gives the stabilizing effect of magnetic field line bending while $-\omega_{D}^{\prime 2}$ gives a destabilizing Kelvin-Helmholtz flow shear effect. The resulting stability criterion is the incompressible version of that derived in [47-49], which generalized the Suydam criterion [8] to include flow. Without magnetic and gravitational fields and density gradients, Eq. (B15) was first obtained in Ref. [30]. A magnetic field was included in this reference only for axisymmetric instabilities $(n=0)$ and pure rotation $\left(u_{z}=0\right)$. This generalized the result of Velikhov [1] whose analysis excluded an azimuthal magnetic field $B_{\phi}$.

\section{APPENDIX C: NEGATIVE ENERGY WAVES}

In Appendix B 1 we took the inner product of Eq. (A3) with $\xi^{*}$. When instead we take the inner product of Eq. (A3) with $\partial \boldsymbol{\xi}^{*} / \partial t$ and use that $\mathbf{F}$ and $-i \rho \mathbf{u} \cdot \boldsymbol{\nabla}$ are self-adjoint operators, we can obtain $\partial E / \partial t=0$. Here, the mode energy $[28,34,35]$

$$
E=\frac{1}{2} \int\left(\rho\left|\frac{\partial \boldsymbol{\xi}}{\partial t}\right|^{2}-\xi^{*} \cdot \mathbf{F}\right) d^{3} \mathbf{r}=\left(|\omega|^{2}+\bar{W}\right) I e^{2 \operatorname{Im}(\omega) t} .
$$

For unstable modes, Eq. (B1) gives $\left|\omega_{ \pm}\right|^{2}=-\bar{W}$ so that $E=$ 0 . For stable modes however, Eq. (B1) gives $\left|\omega_{ \pm}\right|^{2}=\bar{W}+$ $2 \bar{V} \omega_{ \pm}$so that

$$
E=2 \omega_{ \pm}\left(\omega_{ \pm}-\bar{V}\right) I= \pm 2 \omega_{ \pm} I \sqrt{\bar{V}^{2}+\bar{W}} .
$$

This shows that when $\omega_{+}$and $\omega_{-}$have the same sign, the two solutions of Eq. (B1) correspond to a pair of modes with energies of opposite sign. A negative energy mode accompanied by one with positive energy. Note that this is only possible when

$$
0<-\bar{W} \leqslant \bar{V}^{2} .
$$

The stability criterion of Eq. (1) is derived in Ref. [7] under the assumption that the Coriolis shift and the Doppler shifted mode frequency $\omega_{D}=\omega+n \Omega$ vanish. At marginal stability then $\bar{V}^{2}=n^{2} \Omega^{2}=-\bar{W}$, marginally satisfying Eq. (C3). Before the system becomes unstable there will therefore be two modes, one with a small positive energy and one with an opposite negative energy, that combine to form an instability.

The condition $\omega_{D}=0$ implies that the "pattern frequency" $-\omega / n$ locally equals the plasma rotation frequency $\Omega$. This 
co-rotation condition holds quite generally for nonaxisymmetric instabilities [40]. In instabilities like the Papaloizou-Pringle instability [41] or the Rossby wave instability [42], waves reflect at the boundaries or get trapped near a maximum of an equilibrium quantity, respectively. In a magnetized plasma we may speculate that the localizing effect of magnetic field line bending can play a similar role in confining waves with positive and negative energy.
[1] E. P. Velikhov, Sov. Phys. JETP 36, 995 (1959).

[2] S. A. Balbus and J. F. Hawley, Astrophys. J. 376, 214 (1991).

[3] L. J. Zheng, M. S. Chu, and L. Chen, Phys. Plasmas 6, 1217 (1999).

[4] C. Wahlberg and A. Bondeson, Phys. Plasmas 8, 3595 (2001).

[5] F. L. Waelbroeck, Phys. Plasmas 3, 1047 (1996).

[6] C. Wahlberg, Plasma Phys. Control. Fusion 51, 085006 (2009).

[7] J. W. Haverkort and H. J. de Blank, Plasma Phys. Control. Fusion 53, 045008 (2011).

[8] B. R. Suydam, Proc. Second UN Int. Conf. on Peaceful Uses of Atomic Energy 31, 157 (1959).

[9] C. Mercier, Nucl. Fusion 1, 47 (1960).

[10] A. A. Ware and F. A. Haas, Phys. Fluids 9, 956 (1966).

[11] V. D. Shafranov and E. I. Yurchenko, Sov. Phys. JETP 26, 682 (1968).

[12] E. Frieman and M. Rotenberg, Rev. Mod. Phys. 32, 898 (1960).

[13] I. B. Bernstein, E. A. Frieman, M. D. Kruskal, and R. M. Kulsrud, Proc. R. Soc. London 244, 17 (1958).

[14] J. W. Haverkort, H. J. de Blank, and B. Koren, J. Comput. Phys. 231, 981 (2012).

[15] M. N. Bussac and R. Pellat, Phys. Rev. Lett. 35, 1638 (1975).

[16] H. J. de Blank, Ph.D. thesis, Utrecht University, 1990.

[17] B. van der Holst, A. J. C. Beliën, and J. P. Goedbloed, Phys. Plasmas 7, 4208 (2000).

[18] C. Wahlberg and A. Bondeson, Phys. Plasmas 7, 923 (2000).

[19] V. P. Lakhin, V. I. Ilgisonis, and A. I. Smolyakov, Phys. Lett. A 374, 4872 (2010).

[20] A. J. C. Beliën, M. A. Botchev, J. P. Goedbloed, B. van der Holst, and R. Keppens, J. Comput. Phys. 11, 91 (2002).

[21] J. P. Goedbloed, A. J. C. Beliën, B. van der Holst, and R. Keppens, Phys. Plasmas 11, 28 (2004).

[22] J. W. S. Blokland, B. van der Holst, R. Keppens, and J. P. Goedbloed, J. Comput. Phys. 226, 509 (2007).

[23] F. L. Waelbroeck and L. Chen, Phys. Fluids B 3, 601 (1991).

[24] A. J. Webster and H. R. Wilson, Phys. Rev. Lett. 92, 165004 (2004).

[25] R. Hollerbach, V. Teeluck, and G. Rüdiger, Phys. Rev. Lett. 104, 044502 (2010).

[26] R. Keppens, F. Casse, and J. P. Goedbloed, Astrophys. J. 569, L121 (2002).
[27] C. Wang, J. W. S. Blokland, R. Keppens, and J. P. Goedbloed, J. Plasma Phys. 70, 651 (2004).

[28] J. P. Goedbloed, R. Keppens, and S. Poedts, Advanced Magnetohydrodynamics (Cambridge University Press, Cambridge, UK, 2010).

[29] G. I. Ogilvie and J. E. Pringle, Mon. Not. R. Astron. Soc. 279, 152 (1996).

[30] L. N. Howard and A. S. Gupta, J. Fluid Mech. 14, 463 (1962).

[31] A. B. Mikhailovskii, J. G. Lominadze, R. M. Galvão, A. P. Churikov, N. N. Erokhin, A. I. Smolyakov, and V. S. Tsypin, Phys. Plasmas 15, 052103 (2008).

[32] H. Ren, Z. Wu, J. Cao, C. Dong, and P. K. Chu, Plasma Phys. Control. Fusion 53, 035012 (2011).

[33] T. G. Cowling, Astrophys. J. 114, 272 (1951).

[34] I. V. Khalzov, A. I. Smolyakov, and V. I. Ilgisonis, Phys. Plasmas 15, 054501 (2008).

[35] V. I. Ilgisonis, I. V.Khalzov, and A. I. Smolyakov, Nucl. Fusion 49, 035008 (2009).

[36] J. W. Haverkort, Plasma Phys. Control. Fusion 54, 025005 (2012).

[37] D. Lynden-Bell and J. P. Ostriker, Mon. Not. R. Astron. Soc. 136, 293 (1967).

[38] A. Bhattacharjee, R. Iacono, J. L. Milovich, and C. Paranicas, Phys. Fluids B 11, 2207 (1989).

[39] J. Andries, Phys. Plasmas 17, 112106 (2010).

[40] D. J. Acheson, J. Fluid Mech. 61, 609 (1973).

[41] J. C. B. Papaloizou, Mon. Not. R. Astron. Soc. 208, 721 (1984).

[42] R. V. E. Lovelace, Astrophys. J. 513, 805 (1999).

[43] V. P. Lakhin and E. P. Velikhov, Phys. Lett. A 369, 98 (2007).

[44] R. Hollerbach and G. Rüdiger, Phys. Rev. Lett. 95, 124501 (2005).

[45] F. Stefani, T. Gundrum, G. Gerbeth, G. Rüdiger, M. Schultz, J. Szklarski, and R. Hollerbach, Phys. Rev. Lett. 97, 184502 (2006).

[46] J. P. Goedbloed and S. Poedts, Principles of Magnetohydrodynamics (Cambridge University Press, Cambridge, UK, 2004).

[47] E. Hameiri, Ph.D. thesis, New York University, 1976.

[48] E. Hameiri, J. Math. Phys. 22, 2080 (1981).

[49] A. Bondeson, R. Iacono, and A. Bhattacharjee, Phys. Fluids 30, 2167 (1987) 